\title{
La perspectiva moral de las relaciones de victimización entre iguales: un análisis exploratorio de las atribuciones de adolescentes españoles y portugueses
}

\author{
M $^{\mathrm{a}}$ Jesús Caurcel $^{1}$ y Ana Almeida ${ }^{2}$ \\ ${ }^{1}$ Universidad de Granada (España) \\ ${ }^{2}$ Universidade do Minho (Portugal)
}

En el presente estudio, se exploran las atribuciones de emociones morales de los adolescentes, ante una historia de maltrato entre iguales en el contexto escolar. Con ese objetivo, les preguntamos a cerca de los sentimientos de culpa, vergüenza, orgullo e indiferencia de agresores, víctimas y espectadores y les pedimos que justificaran la atribución de los mismos. Procuramos además saber si en términos de su experiencia personal se identifican o se desvinculan de cualquiera de ellos. Participaron un total de 1.237 preadolescentes y adolescentes españoles y portugueses de edades comprendidas entre los 11 y los 16 años. Para el estudio diseñamos el cuestionario SCAN-Bullying 2005 con soporte gráfico (conjunto de viñetas que representan una historia típica de maltrato entre iguales en el contexto escolar). Los resultados muestran un patrón emocional diferente en función del rol: orgullo e indiferencia fueron las emociones más atribuidas a los agresores, inversamente la vergüenza fue la emoción más atribuida a la víctima, mientras que la indiferencia, vergüenza y culpa se atribuyeron en porcentajes muy semejantes a los espectadores. La investigación se inscribe en el cuadro teórico de estudios anteriores que analizan el fenómeno del agresor feliz y de la desvinculación moral, y a la luz de este encuadramiento teórico, procuraremos discutir los resultados.

Palabras clave: Maltrato entre iguales, emociones morales, razonamiento sociomoral, adolescentes.

The moral perspective of the relations of peer bullying: An exploratory analysis of the attributions of Spanish and Portuguese adolescents. This study explores moral emotions associated to adolescents when discussing about peer bullying in school contexts. Bearing this objective in mind, we asked participants about the emotional experience of guilt, shame, pride, and indifference of bullies, victims and bystanders. In addition, we asked them to justify these associations. Secondly, we analysed whether their personal experience is distinctive from the emotional experience of story's protagonists in any of the abovementioned roles. A total of 1237 Spanish and Portuguese pre-adolescents and adolescents from 11 to 16 year-olds took part in this study. We applied the SCAN-Bullying 2005 questionnaire, with graphic support (drawings depicting a typical story on peer bullying in a school context). Results showed a different emotional profile depending on the role: pride and indifference were mostly associated with bullies; shame was more associated with the victims; and indifference, shame and guiltiness with bystanders. This research is embedded in former studies that analyse the happy victimizer phenomenon and moral disengagement, and in the results are discussed in line with this theoretical framework.

Key words: Peer bullying, moral emotions, sociomoral reasoning, adolescents.

Correspondencia: María Jesús Caurcel Cara. Departamento de Psicología Evolutiva y de la Educación. Facultad de Ciencias de la Educación. Universidad de Granada. Campus de Cartuja s/n, C.P. 18171, Granada (España). E-mail: caurcel@ugr.es 
El predomino de estudios sobre el maltrato entre iguales se acentuó a partir de las preocupaciones centradas en la caracterización de este fenómeno y de sus efectos en el ajuste psicosocial de agresores y víctimas, en particular en el periodo escolar. Indudablemente, estos estudios se evidenciaron determinantes en el plano epidemiológico y de la prevención, y no menos importantes para obtener una visión del fenómeno a escala mundial (Smith et al., 1999; Smith, Cowie, Olaffson, y Liefooghe, 2002). Gracias a estudios transnacionales se realizan análisis comparativas y evolutivas que posibilitan identificar aspectos y factores comunes y diferenciados entre culturas, aquellos que permanecerán sensiblemente idénticos con el pasar del tiempo y otros, que siendo relativamente recientes, pueden ser considerados manifestaciones emergentes.

Pasados cerca de 30 años desde los primeros estudios en que el fenómeno del maltrato entre iguales fue explícitamente referenciado (Olweus, 1977; 1978), la prevalencia del fenómeno y su trayectoria en ambos sexos no conocen alteraciones significativas, mientras que nuevas formas de victimización van siendo asociadas al uso de las nuevas tecnologías de la información como: SMS, chats, mensajes de vídeo, etc (Belsey, 2005; Smith, 2006).

Los estudios realizados también evidencian que el fenómeno de la victimización entre iguales no se limita a una relación de malos tratos entre agresores y víctimas, sino que los procesos de grupo, que los refuerzan y les otorgan sus papeles a los agentes implicados, tienen un efecto determinante en la aparición y la evolución del mismo. Las investigaciones que ofrecen una visión sistémica del maltrato entre iguales precisan que este fenómeno difícilmente podrá ser desligado del contexto del grupo de iguales y de sus características estructurales, en torno a las cuales emergen y se consolidan papeles y patrones de interacción y relacionamiento social (Craig y Pepler; 1997; Almeida, Caurcel y Machado, 2006; Salmivalli, Lagerspetz, Björkqvist, Österman y Kaukiainen, 1996).

La investigación sobre el maltrato entre iguales, aún se revela como un área de gran interés para el estudio de procesos psicológicos de diverso orden. Particularmente cruciales, fueron los estudios enmarcados dentro del paradigma de la cognición social (Dodge, 1991; Kaukiainen, Bjorkqvist, Ostermann, y Lagerspetz, 1996). La evidencia empírica, que estos estudios producirán acerca de los distintos patrones de atribución, posibilitó, entre otros aspectos, la distinción entre agresores proactivos y reactivos y asociar el pensamiento a la acción. Efectivamente, si los primeros se muestran como poseedores de capacidades de planificación, de raciocinio frío, insensibles y hábiles manipuladores, los segundos tienden con frecuencia a distorsionar el significado de las situaciones sociales y a atribuir una intencionalidad negativa a comportamientos 
ambiguos por parte de otros, lo que los lleva a tomar represalias con base a una interpretación evidenciada - venganza que normalmente se caracteriza por respuestas agresivas de fuerte carga emocional (Sutton, Smith y Swettenham, 1999a, 1999b).

Los procesos sociomorales asociados al maltrato entre iguales, por comparación con los cognitivos, han sido menos investigados. Sin embargo, si tomamos en consideración que estamos ante un fenómeno social que ocurre en el seno de un grupo y que se perpetúa debido al refuerzo y la aprobación social que obtienen de algunos elementos del grupo, no caben dudas de que cabría evaluar en qué medida este apoyo transcurre a través de normas socialmente aceptadas y validadas con importantes implicaciones morales.

En el cuadro de la cognición social, para este apoyo concurren factores sociocognitivos específicamente relacionados con aspectos morales. Y si en otros estudios investigamos qué influencia podría tener la percepción y la comprensión que el grupo tiene del fenómeno en sí mismo, del comportamiento de agresores y víctimas, y también de los observadores, y qué sentimientos atribuyen a unos y a otros (Almeida y del Barrio, 2002; del Barrio, Almeida, van der Meulen, Barrios y Gutiérrez, 2003; Almeida et al., 2006), para el presente estudio nos centraremos en la exploración de las atribuciones morales.

Para este efecto analizaremos las emociones morales atribuidas a agresores y víctimas. Este estudio surge de las indagaciones hechas en investigaciones anteriores en las que se registraron en niños y adolescentes una inversión de desarrollo del patrón moral para los agresores. Estos estudios, sobradamente conocidos por el paradigma del victimizador feliz (Nunner-Winkler y Sodian, 1988; Murgatroyd y Robinson, 1993; Séller y Edelstein, 1993; Keller, Lourenço, Malti, Saalbach, 2003; Lourenço, 1998), constataron que las emociones positivas atribuidas a los 'agresores felices' ('happy victimizer') eran justificadas en el interés personal u otros resultados sensiblemente reforzadores. Así, aunque los niños eran conscientes de las obligaciones morales mostraban una transferencia para un plano secundario en el contexto de la acción. Ante esta incongruencia el interés de los resultados fue discutido para distinguir la atribución psicológica simple (p.e. los motivos para la acción o los sentimientos del protagonista) y el conocimientos moral necesario (p.e. lo correcto y moralmente justo) (Lourenço, 1997; $1998 ; 2000 ; 2001 ; 2003)$. En primera instancia, las atribuciones evidenciaban la elección y objetivos para la acción; en segunda, la elección moral de los protagonistas. 
Considerando que la evaluación moral está influenciada por factores motivacionales que aumentan la disposición para sentir culpa, vergüenza, orgullo, empatía o falta de ella, en una situación de maltrato entre iguales estas emociones morales son importantes componente de la voluntad moral y del deseo de transgredir o actuar a favor de la víctima. A este respecto, Blasi (2003) llama la atención para que se distinga el sentimiento de imputabilidad o sentimiento de responsabilidad personal sobre sus actos y la importancia que les está asociada relativamente al concepto de sí mismo como ser moral ('moral self') o si preferimos, en una acepción más Wittgensteiniana, la consciencia moral de sí mismo. Probablemente los juicios morales integrarán más motivos para el comportamiento moral y para la transgresión moral y explorando las diferencias de perspectiva de las personas implicadas en transgresiones sociales podremos descubrir los motivos para la diferenciación de los juicios morales a cerca del comportamiento del otro y de uno mismo.

Así, en el contexto de nuestro estudio se solicitó a los participantes que identificasen la experiencia emocional de diferentes protagonistas de situaciones de maltrato entre iguales. Más allá del interés específico por el estudio de las atribuciones emocionales de hipotéticos agresores y víctimas y de las justificaciones para actuar como tal, también procuraremos observar la relación entre las diferentes experiencias emocionales en los distintos papeles de los participantes en el estudio y de la perspectiva de sí mismo en esos diferentes papeles.

El presente estudio, tiene por objetivo abordar algunos estos aspectos socio-cognitivos y, más concretamente, dilucidar el papel de las emociones morales. Con ese objetivo le preguntamos a adolescentes españoles y portugueses a cerca de los sentimientos de culpa, vergüenza, orgullo e indiferencia de agresores y víctimas. Procuramos además saber si en términos de su experiencia personal se identifican o se desvinculan de cualquiera de ellos. De este modo, la investigación se inscribe en el cuadro teórico de estudios anteriores que analizan el fenómeno del agresor feliz y de la desvinculación moral, y a la luz de este encuadramiento teórico procuraremos discutir los resultados.

\section{METODO}

\section{Participantes}

En este estudio participaron un total de 1.237 preadolescentes y adolescentes, 842 de España y 395 de Portugal, con edades comprendidas entre los 11 y los 16 años (edad media $=13,27)$ y con una distribución prácticamente balanceada en función del sexo (631 chicos y 606 chicas). 
La muestra fue constituida por alumnos de escuelas públicas y privadas representativas de las comunidades urbanas y del medio socioeconómico heterogéneo de las ciudades en las que el estudio fue desarrollado, Granada (España) y Braga (Portugal). Debido a las diferencias entre el sistema educativo de cada país, adolescentes con la misma edad frecuentaban diferentes niveles de enseñanza y fueron seleccionados en escuelas de enseñanza primaria o secundaría obligatoria, según correspondieran al intervalo de edad designado. En las escuelas escogidas, de acuerdo con los criterios de la muestra, procedimos a la selección aleatoria de un número variables de grupos-clase, de acuerdo con la población de la escuela. Dentro de estas, la participación de los alumnos se basó en la voluntad personal de colaborar en el estudio y la obtención del consentimiento personal, parental y de la dirección del centro, atendiendo a las normas éticas para la participación de preadolescentes y adolescentes en estudios de investigación.

\section{Procedimiento}

Tras contactar con el Equipo directivo de las escuelas seleccionadas al azar y obtener su consentimiento para realizar el estudio, la recogida de datos se llevó a cabo durante los meses de mayo y junio de 2005, ya que, al tratarse del final del curso académico, los alumnos se conocerían mejor entre sí. La cumplimentación de los cuestionarios se realizó en una única sesión de dos horas de duración, en el aula, de forma colectiva y contando sólo con la presencia de los investigadores. Con la intención de preservar el anonimato y garantizar la completa libertad de los participantes, para expresar su propio pensamiento, se les pidió que rellenaran un recuadro en la esquina superior derecha de los cuestionarios con su nombre real y con un nick o nombre ficticio con el que se les identificaría en adelante. El recuadro era recortado por los investigadores en presencia de los participantes y guardado en un sobre.

\section{Instrumentos}

Test de Evaluación de Agresividad entre Escolares (BULL-S) (Cerezo, 2000). Es un instrumento sencillo basado en la técnica del sociograma, diseñado con la finalidad de analizar y comprender la dinámica de agresión y victimización en el medio escolar. Presenta dos modalidades: la Forma A (colectiva), para alumnos entre 7 y 16 años, que es la que se ha utilizado, y la Forma P (individual), para profesores. La modalidad para alumnos nos da información sobre la posición social de cada miembro del grupo y de la estructura del grupo social en su conjunto, así como identificar el papel desempeñado por cada alumno en situaciones de maltrato entre iguales.

Cuestionario SCAN-Bullying 2005 (Almeida y Caurcel, 2005). Es un instrumentos de investigación, que consiste en una narrativa gráfica, presentada en un 
soporte de 10 viñetas representativas de diferentes situaciones de maltrato entre iguales en el contexto escolar y de 5 viñetas adicionales que representan diferentes soluciones para la historia presentada (Almeida et al., 2001; del Barrio et al., 2003). El SCAN-Bullying-2005, sustituye la entrevista clínica del instrumento inicial por un cuestionario que aborda varias cuestiones relacionadas con el maltrato entre iguales, que cubren las percepciones y los significados, las actitudes y sentimientos atribuidos a los protagonistas de la historia y la perspectiva de los propios adolescentes en el papel de agresores, víctimas y observadores. Se han construido dos versiones del instrumento: femenina y masculina, y en castellano y en portugués.

Figura 1. Ejemplos de algunas viñetas del SCAN-Bullying, versión femenina y masculina
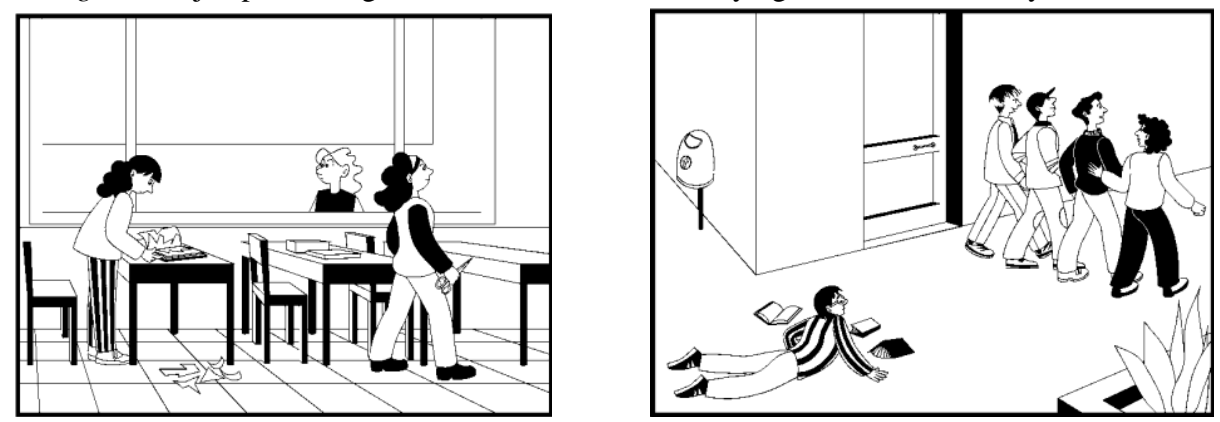

El cuestionario consta de 36 ítems y está organizado en diferentes secciones:

a) Datos personales del participante.

b) Representación del fenómeno.

c) Tipos de maltrato.

d) Causalidad.

e) Bloque de preguntas sobre la víctima: perfil de la víctima; emociones generales que ellos atribuyen a la víctima y que ellos sentirían si fueran la víctima; estrategia de afrontamiento si él fuera ese chico; experiencia como víctima.

f) Experiencia como espectador.

g) Bloque de preguntas sobre el grupo agresor: perfil del agresor; emociones generales que ellos atribuyen al agresor/es y que ellos sentirían si fueran el agresor; experiencia como agresor.

h) Atribución de emociones morales a los personajes.

i) Final de la historia.

j) Perfil del participante. 


\section{RESULTADOS}

Los datos obtenidos fueron analizados estadísticamente utilizando el programa SPSS (Statistical Package for Social Science) en su versión 15.0.

Se pidió a los participantes que respondieran a las siguientes preguntas:

Desde tu punto de vista, ¿cómo se siente/n este chico/s?: culpable/s, avergonzado/s, orgulloso/s y/o desinteresado/s (se les indicó a través de una viñeta a qué personaje/s de la historia nos referíamos). Esta cuestión iba destinada a identificar las atribuciones de los participantes para las diferentes emociones morales (culpa, vergüenza, orgullo e indiferencia) a los distintos personajes de la historia. Para ello, debían señalar si la emoción fue atribuida a no al personaje, utilizando una escala dicotómica si/no. También se les pidió que trataran de justificar esas atribuciones, presentándoles una serie de pensamientos al respecto y pidiéndoles que respondieran qué personaje creían que pensaba de esa manera.

¿Cómo te sentirías si tú fueses este chico/s?: culpable/s, avergonzado/s, orgulloso/s y/o desinteresado/s (de nuevo se les indicó a través de una viñeta a qué personaje/s de la historia nos referíamos). Esta cuestión iba destinada a determinar, si en términos de su experiencia personal, se identifican o se desvinculan de cualquiera de los personajes. Al igual que en la pregunta anterior, debían señalar sí sentirían o no esa/s emociones, en función del papel que asumieran utilizando una escala dicotómica si/no.

Para conocer la distribución de los resultados se calcularon los estadísticos descriptivos y las frecuencias (Tabla 1) y para comprobar la existencia de diferencias significativas entre las emociones morales atribuidas a los personajes en función de la experiencia personal del participante, se utilizó la prueba no paramétrica test de chi-cuadrado de Pearson $\left(x^{2}\right)$.

Emociones morales atribuidas a la víctima. Como se puede observar en la tabla 1, la vergüenza es la emoción moral más atribuida a la víctima (88\%), justificándola por las consecuencias negativas (psicológicas, sociales, afectivas, escolares...) que sufre con esta situación de maltrato, seleccionado en un $90,2 \%$ la respuesta "Me avergüenzo cuando me humillan en público" $\left(\mathrm{x}^{2}=28,299, \mathrm{p}=, 00<, 01\right)$, o bien debido al propio comportamiento exhibido por la víctima seleccionando la opción "Debo haber hecho algo para que me hagan esto" $(89,6 \%)\left(x^{2}=13,799, \mathrm{p}=, 00<, 01\right)$. Sin embargo, cuando se pide a los participantes que tomen la perspectiva de la víctima (que asuman el papel de la 
víctima) la atribución de vergüenza $(80,0 \%$ versus $65,3 \%)$ disminuye significativamente $\left(x^{2}=57,492, p=, 00<, 01\right)$.

Tabla 1. Atribución de emociones a los distintos personajes de la muestra en diferentes condiciones.

(Porcentajes, $\mathrm{N}=1.221$ )

\begin{tabular}{c|lc|lr}
\hline Personajes de la historia & Se siente... & & \multicolumn{2}{l}{ Participante se sentiría... } \\
\hline \multirow{2}{*}{ Víctima } & Culpable & $38,4 \%$ & Culpable & $25,1 \% \downarrow$ \\
& Avergonzado & $88,0 \%$ & Avergonzado & $65,3 \% \downarrow$ \\
& Indiferente & $28,8 \%$ & Indiferente & $29,3 \% \uparrow$ \\
& Orgulloso & $6,9 \%$ & Orgulloso & $19,0 \% \uparrow$ \\
\hline \multirow{2}{*}{ Grupo Agresor } & Culpable & $15,6 \%$ & Culpable & $82,6 \% \uparrow$ \\
& Avergonzado & $9,3 \%$ & Avergonzado & $75,5 \% \uparrow$ \\
& Indiferente & $54,5 \%$ & Indiferente & $32,3 \% \downarrow$ \\
& Orgulloso & $89,8 \%$ & Orgulloso & $21,1 \% \downarrow$ \\
\hline \multirow{3}{*}{ Agresor líder } & Culpable & $18,5 \%$ & Culpable & $81,3 \% \uparrow$ \\
& Avergonzado & $14,1 \%$ & Avergonzado & $77,7 \% \uparrow$ \\
& Indiferente & $50,7 \%$ & Indiferente & $27,4 \% \downarrow$ \\
& Orgulloso & $79,6 \%$ & Orgulloso & $16,8 \% \downarrow$ \\
\hline \multirow{2}{*}{ Espectadores } & Culpable & $40,6 \%$ & Culpable & $58,0 \% \uparrow$ \\
& Avergonzado & $43,7 \%$ & Avergonzado & $58,9 \% \uparrow$ \\
& Indiferente & $47,5 \%$ & Indiferente & $24,7 \% \downarrow$ \\
& Orgulloso & $15,9 \%$ & Orgulloso & $11,5 \% \downarrow$ \\
\hline
\end{tabular}

En consonancia con lo anterior, alrededor del $38 \%$ de los participantes atribuyen culpa a la víctima, lo que coincide con la idea de que la víctima es la causa del maltrato a falta de otro tipo de explicación más evidente. Y justifican su atribución, refiriéndose a rasgos, características o comportamientos de la víctima que son difíciles de cambiar eligiendo en un 41,3\% la respuesta "Debo haber hecho algo para que me hagan esto" $\left(\mathrm{x}^{2}=18,937, \mathrm{p}=, 00<, 01\right)$ y en un $41,1 \%$ la opción "Tal vez tengo alguna cosa que no les gusta" $\left(\mathrm{x}^{2}=16,801, \mathrm{p}=, 00<, 01\right)$. Cuando se les pide a los participantes que asuman el papel de la víctima observamos de nuevo que la atribución de culpa disminuye significativamente $(38,4 \%$ versus $25,1 \%)\left(\mathrm{x}^{2}=241,510, \mathrm{p}=, 00<, 01\right)$.

Frente a la atribución de las dos emociones anteriores, llama la atención, que un 28,8\% de los participantes considere que la víctima se encuentra indiferente, esto podría entenderse como un mecanismo de defensa o un escudo que la víctima pone para continuar con su vida a pesar de sufrir el maltrato. Además esta atribución emocional aumenta cuando los participantes asumen el papel de la víctima (28,8\% vs 29,3\%) $\left(x^{2}=102,063, p=, 00<, 01\right)$. Sin embargo, esta emoción no ha sido justificada por los participantes de una manera significativa con ninguna de las opciones presentadas en el cuestionario. 
Por otro lado, son muy pocos los participantes que atribuyen orgullo (6,9\%) a la víctima, y cuando lo hacen tampoco justifican significativamente esta reacción emocional con ninguna de las opciones presentadas. Sin embargo, el resultado más interesante lo encontramos cuando se le pide al participante que asuma el papel de víctima, ya que el porcentaje de adolescentes que se sentirían orgullosos, aumentan significativamente pasando del $6,9 \%$ al $19,0 \%\left(\mathrm{x}^{2}=133,905, \mathrm{p}=, 00<, 01\right)$, lo que podría entenderse como un mecanismo de defensa para proteger la propia autoimagen que tienen de sí mismos.

Este patrón de atribución de emociones morales, nos muestra que los adolescentes conciben a la víctima como pasiva, que no puede controlar la situación, que sufre las consecuencias negativas, y que por tanto el maltrato va a continuar.

Emociones morales atribuidas al grupo de agresores. Las emociones morales de orgullo e indiferencia son las más atribuidas al grupo de agresores, con un porcentaje del $89,8 \%$ y del $54,9 \%$ respectivamente. Los adolescentes justifican la atribución de orgullo haciendo referencia a las consecuencias positivas de diferente índole (social, afectivas, psicológicas,...) que obtienen con la situación de maltrato, a través de las opciones "Me siento bien y fuerte cuando hago esto" $(93,5 \%)\left(\mathrm{x}^{2}=15,205, \mathrm{p}=, 00<, 01\right)$ y "Lo hago sólo para divertirme" (92,2\%) $\left(\mathrm{x}^{2}=5,619, \mathrm{p}=, 017<, 05\right)$. Y justifican la atribución de indiferencia considerando que los agresores no empatizan con los sentimientos y consecuencias que el maltrato tiene para la víctima, eligiendo en un 57,1\% la opción "Me siento bien y fuerte cuando hago esto" $\left(\mathrm{x}^{2}=4,707, \mathrm{p}=, 03<, 05\right)$ y que también minimizan los efectos de sus acciones considerándolo un juego o una diversión, escogiendo la respuesta "Lo hago sólo para divertirme" en un 50,0\% $\left(x^{2}=6,991\right.$, $\mathrm{p}=, 08<, 01)$.

Aunque en un porcentaje mucho menor que las dos emociones anteriores, los adolescentes también atribuyen, en un 15,6\%, culpa al grupo de agresores. Como cabía esperar, la justifican por medio de la asunción de la responsabilidad de sus actos, señalando la opción "Me siento mal con lo que hice" $(21,4 \%)\left(x^{2}=8,643, \mathrm{p}=, 01<, 01\right)$.

Finalmente, son muy pocos los participantes que atribuyen la emoción moral de vergüenza al grupo de agresores $(9,3 \%)$. Esta atribución la justifican por medio de una evaluación moral de la conducta de los agresores (preocupación moral) seleccionando opciones como "Tengo remordimientos de no hacer nada para ayudarlo" (18,9\%) $\left(x^{2}=11,498, \mathrm{p}=, 01<, 01\right)$ y "Me siento avergonzado de lo que hice" $(17,1 \%)\left(x^{2}=11,868\right.$, $\mathrm{p}=, 01<, 01$ ), así como de la existencia de una asunción de responsabilidad para parte de los agresores que le lleva a sentirse avergonzados, a través de la opción "Me siento mal 
con lo que hice" $(13,3 \%)\left(x^{2}=8,643, \mathrm{p}=, 03<, 01\right)$, sería una emoción muy próxima a la culpa.

El análisis de este patrón de atribuciones nos permite concluir que los adolescentes perciben al grupo de agresores como victimizadores felices. Sin embargo, cuando se pide a los participantes que tomen la perspectiva del agresor la atribución de orgullo $(89,8 \%$ versus $32,3 \%)$ e indiferencia $(54,5 \%$ versus $21,1 \%)$ disminuye significativamente $\left(x^{2}=827,434, \mathrm{p}=, 00<, 01\right) \quad\left(x^{2}=13,811, \mathrm{p}=, 00<, 01\right)$ lo que puede demostrar una actitud de rechazo hacia este tipo de comportamientos o simplemente una deseabilidad social en la respuesta.

Emociones morales atribuidas al líder del grupo agresor. Al igual que para el grupo de agresores son las emociones morales de orgullo e indiferencia las más atribuidas al el líder del grupo, con un porcentaje del 79,6\% y del 50,7\% respectivamente. Los participantes justifican la atribución de orgullo haciendo referencia a las consecuencias positivas de diferente índole (social, afectivas, psicológicas,...) que obtienen con la situación de maltrato, a través de las opciones "Me siento bien y fuerte cuando hago esto" $(86,4 \%)\left(x^{2}=47,460, \mathrm{p}=, 00<, 01\right)$ y "Lo hago sólo para divertirme" $(84,6 \%)$ $\left(x^{2}=23,544, \mathrm{p}=, 00<, 05\right)$. Y justifican la atribución de indiferencia considerando que para el líder se trata de un juego o una diversión, minimizando los efectos de sus acciones, escogiendo la respuesta "Lo hago sólo para divertirme" en un 54,8\% $\left(x^{2}=13,174\right.$, $\mathrm{p}=, 00<, 01)$.

Siguiendo con la misma línea lógica de atribuciones, los participantes también atribuyen, al igual que al grupo de agresores, culpa al líder y lo hacen en un mayor porcentaje $18,5 \%$ frente $15,6 \%$, lo que demuestra una mayor difusión de la responsabilidad en el seno del grupo. Y para justificar que el líder sienta esta emoción recurren a muy diversas alternativas, por un lado hacen referencia a la asunción de la autoría moral de sus actos (responsabilidad) señalando las opciones de "Me siento avergonzado de lo que hice" $(34,6 \%)\left(x^{2}=42,647, \mathrm{p}=, 00<, 01\right)$ y "Sé que está mal, pero lo hago porque otros también lo hacen" $(24,1 \%)\left(x^{2}=10,725, \mathrm{p}=, 01<, 01\right)$; por otro a la preocupación moral que le produce su conducta con las opciones de "Me avergüenza no hacer nada para ayudarlo" $(40,0 \%)\left(x^{2}=35,287, \mathrm{p}=, 00<, 01\right)$, "Tengo remordimientos por no hacer nada para ayudarlo" $(35,2 \%)\left(x^{2}=17,637, \mathrm{p}=, 00<, 01\right)$ y "Me siento mal con lo que hice" $(28,9 \%)\left(x^{2}=34,789, \mathrm{p}=, 00<, 01\right)$; y por último, al hecho de que el líder sienta empatía hacia la víctima y se preocupe por los sentimientos y consecuencias que el maltrato tiene para ella, marcando las opción de "Me da remordimiento cuando lo veo triste y solo" $(39,8 \%)\left(x^{2}=33,932, \mathrm{p}=, 00<, 01\right)$. 
Finalmente, la emoción moral menos atribuidos al líder es la de vergüenza, haciéndolo en un 14,1\% frente al 9,3\% para el grupo de agresores. Esta atribución moral la justifican por medio de diversas alternativas: la preocupación moral por sus actos seleccionado opciones como "Me da remordimiento cuando lo veo solo y triste" $(30,1 \%)$ $\left(x^{2}=23,630, \mathrm{p}=, 00<, 01\right)$, "Me avergüenza no hacer nada para ayudarlo" $(28,2 \%)$ $\left(x^{2}=19,740, \mathrm{p}=, 00<, 01\right)$, y "Tengo remordimientos de no hacer nada para ayudarlo" $(26,1 \%)\left(x^{2}=11,262, \mathrm{p}=, 01<, 01\right)$; por las consecuencias sociales negativas que tienen sus actos (ser mal vistos, sanciones, castigos...) a través de la opción "Me daría vergüenza si mis padres y profesores supieran lo que hago" $(16,9 \%)\left(x^{2}=4,267, \mathrm{p}=, 039<, 05\right)$ y finalmente, por la asunción de responsabilidad de sus actos: "Me siento avergonzado de lo que hice" $(26,8 \%)\left(x^{2}=32,745, \mathrm{p}=, 00<, 01\right)$ y "Me siento mal con lo que hice" $(21,1 \%)$ $\left(x^{2}=19,326, \mathrm{p}=, 00<, 01\right)$.

El análisis de este patrón de atribuciones de emociones morales nos permite concluir que los adolescentes perciben al líder del grupo de agresores como victimizadores felices. Sin embargo, cuando se pide a los participantes que tomen la perspectiva del agresor la atribución de orgullo (79,6\% versus 16,8\%) e indiferencia $(50,7 \%$ versus $27,4 \%)$ disminuye significativamente $\left(x^{2}=22,265, \mathrm{p}=, 00<, 01\right)\left(x^{2}=8,129\right.$, $\mathrm{p}=, 04<, 01)$, lo que puede demostrar un rechazo hacia el tipo de conducta exhibida por el agresor, una mayor empatía hacía la víctima y una implicación moral con el fenómeno o simplemente una deseabilidad social en la respuesta.

Emociones morales atribuidas a los espectadores. En cuanto a las emociones morales atribuidas a los espectadores, encontramos que se les asignan en porcentajes similares las emociones de indiferencia $47,5 \%$, vergüenza $43,7 \%$ y culpa $40,6 \%$, lo que refleja una caracterización de los espectadores por parte de los participantes, principalmente como pro-agresores. Sin embargo, aunque la emoción más atribuida es la indiferencia, no parecen encontrar ninguna explicación significativa, de entre las que se le presentaron para justificar este sentimiento.

La atribución de vergüenza es justificada por medio de diversas alternativas: la preocupación moral por los actos que está observando o en los que en mayor o media participa escogiendo las opciones "Tengo remordimiento de no hacer nada para ayudarlo" $(46,5 \%)\left(x^{2}=10,036, p=, 02<, 01\right)$ "Me da remordimiento cuando lo veo triste y solo" $(47,2 \%)\left(\mathrm{x}^{2}=17,934, \mathrm{p}=, 01<, 01\right)$ y "Me avergüenza no hacer nada para ayudarlo" $(47,1 \%)\left(\mathrm{x}^{2}=14,569, \mathrm{p}=, 00<, 01\right)$; por las consecuencias sociales negativas que tienen sus actos (ser mal vistos, sanciones, castigos, convertirse en blanco de los agresores...) seleccionado las opciones "Me daría vergüenza si mis padres y profesores supieran lo que 
hago" $(50,2 \%)\left(\mathrm{x}^{2}=5,867, \mathrm{p}=, 015<, 05\right)$ y "No me meto para que no me hagan lo mismo" $(46,7 \%)\left(x^{2}=9,424, p=, 02<, 01\right)$ y finalmente, por la asumir parte de la responsabilidad de lo ocurrido indicando las opciones "Me siento mal con lo que hice" $(53,2 \%)\left(x^{2}=15,567\right.$, $\mathrm{p}=, 00<, 01)$, "Me siento avergonzado de lo que hice" $(49 \%)\left(\mathrm{x}^{2}=7,648, \mathrm{p}=, 06<, 01\right)$ y "Sé que está mal pero lo hago porque otros también lo hacen" $(48,1 \%)\left(x^{2}=5,254\right.$, $\mathrm{p}=, 022<, 05)$.

De una manera muy similar justifican el sentimiento de culpa en los espectadores. Así, hacen referencia a la preocupación moral por los actos que están observando o en los que en mayor o media participan, marcando las opciones "Tengo remordimiento de no hacer nada para ayudarlo" $(46,5 \%)\left(\mathrm{x}^{2}=10,036, \mathrm{p}=, 02<, 01\right)$, "Me da remordimiento cuando lo veo triste y solo" $(44,8 \%)\left(\mathrm{x}^{2}=17,820, \mathrm{p}=, 00<, 01\right)$ y "Me avergüenza no hacer nada para ayudarlo" $(44,3 \%)\left(x^{2}=18,155, \mathrm{p}=, 00<, 01\right)$; por las consecuencias sociales negativas que tienen sus actos (ser mal vistos, sanciones, castigos, convertirse en blanco de los agresores...) seleccionado las opciones "Me daría vergüenza si mis padres y profesores supieran lo que hago" $(47,92 \%)\left(x^{2}=7,622, \mathrm{p}=, 06<, 01\right)$ y "No me meto para que no me hagan lo mismo" $(43,17 \%)\left(\mathrm{x}^{2}=7,096, \mathrm{p}=, 08<, 01\right)$ y finalmente, por asumir parte de la responsabilidad de lo ocurrido indicando las opciones "Me siento mal con lo que hice" $(48,4 \%)\left(x^{2}=10,711, p=, 00<, 01\right)$, "Sé que está mal pero lo hago porque otros también lo hacen" $(48,1 \%)\left(\mathrm{x}^{2}=5,254, \mathrm{p}=, 022<, 05\right)$ y "Me siento avergonzado de lo que hice" $(47,1 \%)\left(\mathrm{x}^{2}=11,710, \mathrm{p}=, 00<, 01\right)$.

Finalmente, la emoción menos atribuida a los espectadores es el orgullo, señalada en un 15,9\% y justificada por las consecuencias positivas que obtendrían con la situación de maltrato, indicando la opción "Me siento bien y fuerte cuando hago esto" $(31,2 \%)\left(x^{2}=17,417, p=, 00<, 01\right)$ o restándole importancia a sus actos (minimización) a través de la opción "Lo hago sólo para divertirme" $(23,1 \%)\left(x^{2}=5,154, p=, 023<, 05\right)$.

Cuando se pide a los participantes que asuman el papel de los espectadores la vergüenza y la culpa aumentan significativamente, pasando de $40,6 \%$ a $58,0 \%$ $\left(\mathrm{x}^{2}=179,279, \mathrm{p}=, 00<, 01\right)$ y de $43,7 \%$ a $58,9 \%\left(\mathrm{x}^{2}=85,573, \mathrm{p}=, 00<, 01\right)$, mientras que la indiferencia y el orgullo disminuyen significativamente, pasando de $47,5 \%$ a $24,7 \%$ $\left(x^{2}=843,491, p=, 00<, 01\right)$ y de $15,9 \%$ a $11,5 \%\left(x^{2}=148,876, p=, 00<, 01\right)$. Esto denota una asunción de responsabilidad en el fenómeno, así como, una implicación moral en el mismo. 


\section{DISCUSION}

En este estudio se ha realizado un análisis exploratorio para conocer el patrón de atribución de emociones morales de los preadolescentes y adolescentes para cada uno de los agentes implicados en situaciones de maltrato entre iguales: víctimas, agresores y espectadores. Globalmente, los resultados revelan que los participantes reconocen la complejidad de la experiencia emocional adscribiendo emociones de distanciamiento y de responsabilidad moral a los diferentes personajes de la historia y para sí mismos en los diferentes papeles.

El patrón emocional fue distinto para cada uno de los roles, especialmente entre las víctimas y los agresores, mostrando su capacidad para percibir la existencia de conflicto y sentimientos mixtos y de articular con lógica las justificaciones a las atribuciones realizadas. Esto muestra, que los adolescentes son intuitivos analistas socioemocionales. Los juicios de los adolescentes estuvieron en consonancia con los de estudios clásicos (Arsenio, 1988; Arsenio y Lover, 1995; Bandura, Barbaranelli, Caprara y Pastorelli, 1996; Keller y Edelstein, 1993; Smetana, 1990; Turiel, 1983) y pueden, en este sentido, aportar nueva evidencia empírica a estos estudios.

Los resultados también confirman la tesis del "víctimizador feliz", en cuanto fenómeno emocional en el que las emociones positivas se superponen a las negativas, en el que las emociones de distanciamiento moral superan a las de responsabilidad. Estas atribuciones de orgullo e indiferencia, se fundamentan en justificaciones denunciadoras de la falta de compromiso moral en el acto de maltratar al otro. Este estado emocional les da poder, refuerza su estatus en el grupo e inhibe los deseos de terminar con el maltrato.

Sin embargo, cuando los participantes atribuyen culpa y vergüenza a los agresores, asumen un entorno de evaluación sociomoral, pudiendo considerar en la concepción de Blasi (2003) que los motivos de esas atribuciones, reflejan la imputabilidad moral vinculada al conocimiento de los principios morales que condenan los actos de maltrato.

Por otro lado, los resultados coinciden con los de estudios previos (Almeida et al., 2004; Berdondini y Dondi, 1999; Colléll y Escudé, 2004, Lewis, 1992; Menesini, 1999) y muestran que los participantes consideran que la víctima experimenta sentimientos de verguienza, autocompasión y autoculpa. Estos estados emocionales que casan con la ansiedad, inseguridad y baja autoestima, los síntomas depresivos y tendencias suicidas que muchos experimentan (Alsaker y Olweus, 1993; Cammack- 
Barry, 2005; Roland, 2002) y con unas estrategias pasivas de afrontamiento del conflicto, de aislamiento y evitación, por lo que no logran cerrar el ciclo de la victimización. Es decir, perciben a la víctima como pasiva, incapaz de controlar la situación, que sufre las consecuencias negativas y que, por tanto, el maltrato va a continuar.

En cuanto a los espectadores, parecen ser identificados como pro-agresores, ya que les atribuyen indiferencia, pero también culpa y vergüenza, revelando el importante papel que éstos juegan en la perpetuación del fenómeno. El que los otros compañeros estén indiferentes ante lo ocurrido, es percibido por la víctima y por los agresores, como una muestra de que la situación goza de la aprobación social del grupo de iguales. Esto naturaliza el acto, dejando aún más sola y desprotegida a la víctima, y apoyando el tipo de comportamiento de los agresores. Por esto, solamente si los espectadores experimentan emociones como la culpa, van a poder internalizar su responsabilidad, van a poder empalizar con la víctima y los va a motivar a ir en la dirección moral y prosocial.

Los resultados son consistentes con estudios previos, que muestran, que cuando se juzga una conducta bajo la perspectiva de uno mismo y desde la de los otros, los participantes tienden a verse a sí mismos de una forma más positiva (Keller et al, 2003).

Finalmente, conviene resaltar la utilidad que estos resultados tienen como base empírica, para diseñar e implementar una adecuada intervención a través de programas, que consideren la dimensión socioemocional del maltrato entre iguales. Esto es especialmente relevante, dado que los aspectos emocionales y sociomorales carecen de peso en el currículum escolar y los maestros y profesores tienen amplias lagunas formativas en estas áreas.

\section{REFERENCIAS}

Almeida, A. y Caurcel, M. (2005). Questionário Scan-Bullying. Versão Portuguesa e Castelhana. Registo de obra na Inspecção-Geral das Actividades Culturais, Ministério da Cultura.

Almeida, A., Caurcel, M.J. y Machado, J. (2006). Perceived characteristics of victims according to their victimized and nonvictimized peers. Electronical Journal of Research in Educational Psychology, 9(4), 371-396.

Almeida, A. y del Barrio, C. (2002). A vitimização entre companheiros em contexto escolar. En C. Machado y R. Gonçalves (Coords.), Violência e vítimas de crimes. Crianças. Vol. 2 (pp. 169-197). Coimbra: Quarteto. 
Almeida, A., del Barrio, C., Lisboa, C., van derMuelen, K., Barrios, A., Gutiérrez, H., Granizo, L. y Caurcel, M.J. (2004). They should feel guilty, but they don't ...": adolescents' moral reasoning in bullying relations. Poster presentado en 18th Biennial Meeting of the International Society for the Study of Behavioural Development (ISSBD), Ghent, (Bélgica).

Almeida, A., del Barrio, C., Marques, M., Fernández, I., Gutiérrez, H. y Cruz, J. (2001). A script-cartoon narrative of peer-bullying in children and adolescents. En M. Martínez (Ed.), Prevention and control of aggression and its impact on its victims (pp. 161-168). New York: Kluwer Academic/ Plenum Publishers.

Alsaker, F.D. y Olweus, D. (1993). Global self-evaluations and perceived instability of self in early adolescence: A cohort longitudinal study. Scandinavian Journal of Psychology, 34, 47-63.

Arsenio, W. (1988). Children's conceptions of the situational affective consequences of sociomoral events. Child Development, 59, 1611-1622.

Arsenio, W. y Lover, A. (1995). Children's conceptions of sociomoral affect: happy victimizers, mixed emotions, and other expectancies. En M. Killen y L. Nucci (Eds.), Morality in everyday life: Developmental perspectives (pp. 87-128). Cambridge: Cambridge University Press.

Bandura, A., Barbaranelli, C., Caprara, G.V. y Pastorelli, C. (1996). Mechanisms of Moral Disengagement in the exercise of Moral Agency. Journal of Personality and Social Psychology, 77(2), 364-374.

Belsey, B. (2005) Cyberbullying: An emerging Threta to the always on generation.

Berdondini, L. y Dondi, M. (1999). How do bullies, victims and bystanders react during a session of group work? Comunicación presentada en la IX European Conference on Developmental Psychology, Spetses (Grecia).

Blasi, A. (2003). The developmental of responsibility in children and adolescents. The sense of accountability. Ponencia presentada en la XIth European Conference on Developmental Psychology, Milán, Agosto, 25.

Cammack-Barry, T. (2005). Long-term impact of elementary school bullying victimization on adolescents. Dissertation Abstracts International: Section B: The Sciences and Engineering, 65 (9-B), 4819. 
Cerezo, F. (2000). BULL-S. Test de Evaluación de la Agresividad entre Escolares. Bizcaia: Grupo ALBO-COHS.

Collell, J. y Escudé, C. (2004), Rol de les emocions en els processos de maltractament entre alumnes. Àmbits de Psicopedagogia, 12, 21-26.

Craig, W. y Pepler, D. (1997). Observations of Bullying and Victimization in the School Yard. Canadian Journal of School Psychology, 13(2), 41-60.

Del Barrio, C., Almeida, A., van der Meulen, K., Barrios, A. y Gutiérrez, H. (2003). Representaciones acerca del maltrato entre iguales, atribuciones emocionales y percepción de estrategias de cambio a partir de un instrumento narrativo: SCAN- Bullying. Infancia y Aprendizaje, 26, 63-78.

Dodge, K.A. (1991). The structure and function of reactive and proactive aggression. En D. J. Pepler and K. H. Rubin (Eds.) The development and treatment of childhood aggression. Hillsdale, NJ: Erlbaum.

Kaukiainen, A., Björkqvist, K., Österman, K. y Lagerspetz, M.J. (1996). Social intelligence and empathy as antecedents of different types of aggression. In C.F. Ferris and T. Grisson (Eds.), Understanding aggressive behavior in children (pp. 364-366). Annals of the New York Academy of Sciences, 794.

Keller, M. y Edelstein, W. (1993). The development of the moral self from childhood to adolescence. In G.G. Noam y T.E. Wren (Eds.), The moral self (pp. 310-336). Cambridge, MA: The MIT Press.

Keller, M., Lourenco, O., Malti, T. y Saalbach, H. (2003). The multifaceted phenomenon of 'happy victimizers': A cross-cultural comparison of moral emotions. British Journal of Developmental Psychology, 21(1), 1-18.

Lewis, M. (1992). Shame: the exposed self. New York: Free Press.

Lourenço, O. (1997). Children's attributions of moral emotions to victimizers: Some data, doubts, and suggestions. British Journal of Developmental Psychology, 15(4), 425-438.

Lourenço, O. (1998). Transgressores felizes e infelizes na compreensão de emoções morais na criança. Revista de Psicologia, Educação e Cultura, 2, 241-261.

Lourenço, O. (2000). The aretaic domain and its relation to the deontic domain in moral reasoning. In M. Laupa (Ed.), Rights and wrongs: How children and young adults evaluate the world (pp. 46-61). San Francisco: Jossey-Bass.

Lourenço, O. (2001). Understanding the moral meaning of children's attribution of emotions to victimizers. Archives de Psychologie, 69, 65-81. 
Lourenço, O. (2003). Making sense of Turiel's dispute with Kohlberg: The case of the child's moral competence. New Ideas in Psychology, 21, 43-68.

Menesini, E. (1999). Bullying and emotions. En Nature and Prevention of Bullying, TMR Network Project.

Murgatroyd, S.J. y Robinson, E.J. (1993). Children's Judgements of Emotion Following Moral Transgression. International Journal of Behavioral Development, 16(1) 93-111.

Nunner-Winkler, G. y Sodian, B. (1988). Children's understanding of moral emotions. Child Development, Lafayette, 59, 1323-1338.

Olweus, D. (1977). Aggression and peer acceptance in adolescent boys: Two short-term longitudinal studies of ratings. Child Development, 48, 13011313

Olweus, D. (1978). Aggression in the Schools: Bullies and whipping boys. Washington DC: Hemisphere.

Roland, E. (2002) Bullying, depressive symptoms and suicidal thoughts. Educational Research, 44(1), 55 - 67.

Salmivalli, C., Lagerspetz, K.M.J., Björkqvist, K., Österman, K. y Kaukiainen, A. (1996). Bullying as a group process: Participant roles and their relations to social status in the group. Aggresive Behavior, 22, 1-15.

Smetana, J. (1990). Morality and conduct disorders. En M. Lewis and S. Miller (Eds.), Handbook of Developmental Psychopathology (pp. 157-179). New York: Pleneum.

Smith, P.K. (2006). Ciberacoso: naturaleza y extensión de un nuevo tipo de acoso dentro y fuera de la escuela. Ponencia presentada en el Congreso de Educación, Palma de Mallorca.

Smith, P. K., Morita, Y., Junger-Tas, J., Olweus, D., Catalano, R. y Slee, P. (1999). The Nature of School Bullying. A cross-national perspective. London: Routledge.

Smith, P.K., Cowie, H., Olafsson, R. y Liefooghe A. (2002). Definitions of bullying: a comparison of terms used, and age and sex differences, in a 14 country international comparison. Child Development, 73(4), 1119-1133.

Sutton, J., Smith, P.K. y Swettenham, J. (1999a). Social cognition and bullying; Social inadequacy or skilled manipulation? British Journal of Developmental Psychology, 17, 435-450. 
CAURCEL y ALMEIDA. La perspectiva moral de las relaciones de victimización entre iguales

Sutton, J., Smith, P.K. y Swettenham, J. (1999b). Bullying and theory of mind: A critique of the social skills deficit view of anti social behaviour. Social Development, 8, 117-127.

Turiel, E. (1983). The development of social knowledge: Morality and convention. Cambridge: Cambridge University Press.

Recibido: 15 octubre 2007 Modificado: 3 enero 2008 Aceptado: 10 enero 2008 